\title{
Nadler's Theorem on Incomplete Modular Space
}

\author{
Fatemeh Lael $^{1}\left(\mathbb{D}\right.$, Naeem Saleem $^{2} \mathbb{D}$, Liliana Guran ${ }^{3}\left(\mathbb{D}\right.$ and Monica Felicia Bota ${ }^{4, *(\mathbb{D})}$ \\ 1 Imam Khomeini International University-Buin Zahra Higher Education Center of Engineering and \\ Technology, Qazvin 3451866391, Iran; Lael@bzeng.ikiu.ac.ir \\ 2 Department of Mathematics, University of Management and Technology C-II, Johar Town, \\ Lahore 54770, Pakistan; naeem.saleem2@gmail.com \\ 3 Department of Pharmaceutical Sciences, "Vasile Goldiş” Western University of Arad, L. Rebreanu Street, \\ No. 86, 310048 Arad, Romania; guran.liliana@uvvg.ro \\ 4 Department of Mathematics, Babeş-Bolyai University, M. Kogălniceanu Street, No. 1, \\ 400084 Cluj-Napoca, Romania \\ * Correspondence: monica.bota@ubbcluj.ro
}

Citation: Lael, F.; Saleem, N.; Guran, L.; Bota, M. Nadler's Theorem on Incomplete Modular Space. Mathematics 2021, 9, 1927. https:/ / doi.org/10.3390/math9161927

Academic Editors: Yeol-Je Cho, Sang-Eon Han and Jong-Kyu Kim

Received: 2 July 2021

Accepted: 10 August 2021

Published: 13 August 2021

Publisher's Note: MDPI stays neutral with regard to jurisdictional claims in published maps and institutional affiliations.

\begin{abstract}
This manuscript is focused on the role of convexity of the modular, and some fixed point results for contractive correspondence and single-valued mappings are presented. Further, we prove Nadler's Theorem and some fixed point results on orthogonal modular spaces. We present an application to a particular form of integral inclusion to support our proposed version of Nadler's theorem.
\end{abstract}

Keywords: fixed points; incomplete modular space; Nadler's fixed point theorem; integral inclusion

\section{Introduction}

The fixed point results in modular spaces have several applications in various branches of sciences. These results strongly depend on some assumptions which are more theoretic and have no applications in normed vector spaces. Some recent research trends are dedicated to the study of the well-known fixed point theorems by relaxing the assumptions and considering the case of modular spaces.

S. Banach became one of the pioneers of fixed point theory by proving the well known Banach contraction principle in 1922, see [1]. Fixed point theory plays an important role both in the field of mathematics and in applied sciences. Fixed point theory in modular spaces had been considered a starting point in the research field, after being identified as a generalization of normed spaces. See [2-4]. The fixed point theorems in modular spaces presented in the literature deal with rigorous statements and proofs of many interesting problems that give some applications in a wide variety of fields, including machine learning, programming, quantum mechanics, etc.

Fixed point theory in modular space has deep roots (see [5]) Khamsi used some constructive techniques for single-valued mappings to obtain the solutions of some fixed point problems. This work is appreciated by several researchers, and it became an inspiration for a variety of fixed point results. For details see [6,7]. These ideas were extended and generalized by several authors in a variety of ways. One successful approach is that of Nadler, who in 1969, proposed the Banach contraction principle for multivalued mappings in modular spaces [8]. Several authors extended and generalized the results by relaxing the strong assumptions used in (for details, see [9-12]). Furthermore, Latif et al. (in [13]) focused on a particular case for multivalued mappings in modular spaces with the additivity of the modular. Later, the authors (in [14]) explored the existence of fixed points of a specific type of $G$-contractive and $G$-nonexpansive mappings in modular function spaces.

Eshaghi et al. [15] introduced quite recently, the notion of orthogonal set and obtained an extension of Banach's fixed point theorem. They proved, providing an strong example, 
that their main result is a good generalization of Banach's fixed point theorem. After that in [16], orthogonal modular space is defined and a new generalized modular version of the Meir-Keeler fixed point theorem with respect to an orthogonal relation is presented. Nguyen Van Dung, in [17], talked about the importance of the results proved on orthogonal set and showed that many existence results on fixed points in orthogonal complete metric spaces can be proved by using the corresponding existence results in complete metric spaces. For more information about orthogonal space, we refer to [15,18-20].

The aim of this paper is to prove some generalizations of fixed point results in an orthogonal modular space by relaxing some strong assumptions for the modular spaces case such as convexity, continuity and Fatou property.

This paper starts with a brief introduction into modular spaces and orthogonal modular spaces theory along with the relevant literature. Section 3 presents some techniques and methods of improving some known fixed point results. An application to integral inclusions is provided in the last section.

\section{Notations and Background}

This section can be considered as an introduction to fundamental concepts of modular spaces and orthogonal modular spaces. For detailed information see [3,21-28].

\subsection{Modular Spaces}

Consider $X$ a real linear space and $\rho$ a real valued functional on $X$ which satisfies the following conditions:

1. $\rho(x)=0$ if and only if $x=0$,

2. $\rho(-x)=\rho(x)$,

3. $\rho(\alpha x+\beta y) \leq \rho(x)+\rho(y)$, for any $\alpha, \beta \in \mathbb{R}^{\geq 0}$ with $\alpha+\beta=1$.

The functional $\rho$ is called a modular on $X$ and $(X, \rho)$ is called a modular space. A modular induces a vector space $X_{\rho}=\{x \in X: \rho(\alpha x) \rightarrow 0$ as $\alpha \rightarrow 0\}$ which is called a modular linear space. Musielak and Orlicz in $[2,29,30]$ provided the definitions of the following key concepts in a modular space $(X, \rho)$ :

Definition 1. A sequence $\left(x_{n}\right)$ in $B \subseteq X$ is said to be $\rho$-convergent to a point $x \in B$ if $\rho\left(x_{n}-\right.$ $x) \rightarrow 0$ as $n \rightarrow+\infty$.

Definition 2. A $\rho$-closed subset $B \subseteq X$ is a set which has the limits of all its $\rho$-convergent sequences.

Definition 3. A sequence $\left(x_{n}\right)$ in $B \subseteq X$ is named to be $\rho$-Cauchy if $\rho\left(x_{m}-x_{n}\right) \rightarrow 0$ as $m, n \rightarrow+\infty$.

Definition 4. A subset $B$ of $X$ is said to be $\rho$-complete if each $\rho$-Cauchy sequence in $B$ is $\rho-$ convergent to a point of $B$.

Definition 5. $\rho$-bounded subsets: $A$ subset $B \subseteq X_{\rho}$ is called $\rho$-bounded if $\sup _{x, y \in B} \rho(x-y)<+\infty$.

Definition 6. $\rho$-compact subsets: $A \rho$-closed subset $B \subseteq X$ is called $\rho$-compact if any sequence $\left(x_{n}\right) \in B$ has a $\rho$-convergent subsequence.

For a modular space $(X, \rho)$, the function $\omega_{\rho}$ which is said growth function [31] is defined on $[0,+\infty)$ as follows:

$$
\omega_{\rho}(t)=\inf \{\omega: \rho(t x) \leq \omega \rho(x): x \in X, 0<\rho(x)\} .
$$


It is clear that whenever $(X, \rho)$ satisfying $\omega_{\rho}(2)<+\infty$, then every $\rho$-convergent sequence in $(X, \rho)$ is $\rho$-Cauchy. Also, we note that in this case every $\rho$-compact set is a $\rho$-bounded and $\rho$-complete set [32].

We recall that for any subset $C$ of $(X, \rho)$ a correspondence $f$ on a set $C$, denoted by $f: C \rightarrow C$ assigns to each $a \in C$ a (nonempty) subset $f(a)$ of $C$ and if $a \in f(a)$ for some $a \in C$, then $a \in C$ is said to be a fixed point. Also, it is called weakly Picard, if there exists a sequence $\left(x_{n}\right)$ with $x_{0} \in C, x_{1} \in f\left(x_{0}\right)$ and $x_{n+1} \in f\left(x_{n}\right)$ for which is convergent to a fixed point of $f$. For a correspondence $f$, we define $\operatorname{dist}_{\rho}(a, f(b))=\inf \{\rho(a-y): y \in f(b)\}$. Also, Hausdorff distance is defined as follows

$$
H_{\rho}(A, B)=\max \left\{\max _{a \in A} \operatorname{dist}_{\rho}(a, B), \max _{b \in B} \operatorname{dist}_{\rho}(A, b)\right\},
$$

where $A$ and $B$ are subsets of $C$.

\subsection{Orthogonal Modular Spaces}

Now, we recall some definitions on orthogonal set and orthogonal modular space (for more details see [33-36]).

Definition 7. Let $B \neq \varnothing$ and $\perp \subset B \times B$ be a binary relation. If $\perp$ satisfies the following condition there exists $x_{0} \in B ;\left(\left(\right.\right.$ for all $\left.x \in B ; x \perp x_{0}\right)$ or (for all $\left.\left.x \in B ; x_{0} \perp x\right)\right)$,

(where $x_{0}$ is called an orthogonal element). Then B is called an orthogonal set (briefly O-set) which is denoted by $(B, \perp)$.

Definition 8. Let $(X, \rho)$ be a modular space and $(X, \perp)$ be an $O$-set. Then $(X, \rho, \perp)$ is called an orthogonal modular space.

Definition 9. A sequence $\left(x_{n}\right), n \in \mathbb{N}$ is called an orthogonal sequence (briefly, O-sequence) if

$$
\text { ((for all } \left.\left.\left.n ; x_{n} \perp x_{n+1}\right) \text { or (for all } n ; x_{n+1} \perp x_{n}\right)\right) \text {. }
$$

Also it is called a strongly orthogonal sequence (briefly, SO-sequence) if

$$
\left(\left(\text { for all } n, k ; x_{n} \perp x_{n+k}\right) \text { or }\left(\text { for all } n, k ; x_{n+k} \perp x_{n}\right)\right) \text {. }
$$

It is clear that every orthogonal modular space is a modular space, so all Definitions D1-D6 can be defined for $(X, \rho, \perp)$. If a sequence $\left(x_{n}\right)$ is $O$-sequence (SO-sequence) then D7D9 in $(X, \rho, \perp)$ can be defined as $\rho$-convergent $O$-sequence ( $\rho$-convergent $S O$-sequence), $\mathrm{O}$-closed set (SO-closed set), Cauchy $\mathrm{O}$-sequence (Cauchy $\mathrm{SO}$-sequence), respectively.

Definition 10. Let $(X, \rho, \perp)$ (shortly $X)$ be an orthogonal modular space:

a. Then $\mathrm{X}$ is said to be O-complete (SO-complete) if every Cauchy O-sequence (Cauchy SOsequence) is convergent.

Clearly, every O-complete is SO-complete but if X is SO-complete then it is not necessary to be O-complete. Also, there are O-complete spaces which are not complete.

b. Let $B \subset X$. A mapping $T: B \rightarrow B$ is called:

(i) Orthogonal preserving mapping if $x \perp y$ implies $T(x) \perp T(y)$.

(ii) O-continuous (SO-continuous) at $x \in B$ if $T\left(x_{n}\right) \rightarrow T(x)$, for each O-sequence (SOsequence) $x_{n} \in B$ which $x_{n} \rightarrow x$. Also, $T$ is O-continuous (SO-continuous) on B if T is O-continuous (SO-continuous) in each $x \in B$.

Definition 11. Let $(X, \rho, \perp)$ be an orthogonal modular space and $B \subset X$. A correspondence $f: B \rightarrow B$ is called: 
(i) Orthogonal preserving mapping if $x \perp y$ implies $z \perp w$, for every $z \in f(x)$ and $w \in f(y)$.

(ii) Suppose that for any $x \in B$, any $O$-sequence (SO-sequence) $\left(x_{n}\right) \in B$ converging to $x$, and any sequence $\left(y_{n}\right)$ in $B$ such that $y_{n} \in f\left(x_{n}\right)$ for each $n$, there is a $z \in f(x)$ and $a$ subsequence of $\left(y_{n}\right)$ that converges to $z$. Then $f$ is called orthogonal upper hemicontinuous (strongly orthogonal upper hemicontinuous) and denoted as OUHC (SOUHC) at $x \in B$.

\subsection{Relevant Literature}

Fixed point results for a single-valued mapping in modular spaces have been given in $[9,37,38]$. Over the years, many authors have studied some conditions for proving the existence of fixed points for some classes of functions in modular spaces. The Hypotheses (H1)-(H4) are declared as ones of the most common and popular conditions in modular spaces:

Hypothesis 1 (H1). $\Delta_{2}$-condition [29,30]: A modular $\rho$ is said to satisfy the $\Delta_{2}$-condition, if $\rho\left(2 x_{n}\right) \rightarrow 0$, whenever $\sup _{n} \rho\left(x_{n}\right) \rightarrow 0$ as $n \rightarrow+\infty$.

Hypothesis $2(\mathrm{H} 2) . \Delta_{2}$-type condition [29,30]: A modular $\rho$ is said to satisfy the $\Delta_{2}$-type condition if there exists $k>0$ such that $\rho(2 x) \leq k \rho(x)$ for all $x \in X_{\rho}$.

Hypothesis 3 (H3). $\tilde{s}$-convex modular [39]: If condition (3) in the modular definition is replaced by $\rho(\alpha x+\beta y) \leq \alpha^{\tilde{s}} \rho(x)+\beta^{\tilde{s}} \rho(y)$ for all $\alpha, \beta \in[0,+\infty)$ with $\alpha^{\tilde{s}}+\beta^{\tilde{s}}=1$ with an $\tilde{s} \in(0,1]$, the modular $\rho$ is called an $\tilde{s}$-convex modular. In particular, a 1-convex modular is simply called convex.

Hypothesis 4 (H4). Fatou property [40]: A modular $\rho$ has the Fatou property if $\rho(x) \leq$ $\liminf \rho\left(x_{n}\right)$, whenever $x_{n} \rightarrow x$.

Some explicit overviews of $(\mathrm{H} 1)-(\mathrm{H} 4)$ conditions are given in $[5,28,39]$. It is proven that a modular $\rho$ implies that

$$
\|x\|_{\rho}=\inf \left\{a>0: \rho\left(\frac{x}{a}\right) \leq 1\right\}
$$

i.e., it defines an $F$-norm on $X_{\rho}$. Moreover, if $\rho$ is convex, $\|\cdot\|_{\rho}$ is a norm and it is frequently called the Luxemburg norm [41]. Note that a modular space determined by a function modular $\rho$ will be called a modular function space and will be denoted by $L_{\rho}$. Then, it is not difficult to prove that $\|\cdot\|_{\rho}$ is an $F$-norm induced by $\rho$. Then, $\left(L_{\rho},\|\cdot\|_{\rho}\right)$ is a complete space.

Since we are able to define such norm in a real vector space can lead to a simple proof for many fixed point theorems in a specific modular spaces. For example, an earlier work on this topic recall Theorem $2-2$ of [5] which was proposed in the early 1990s.

Theorem 1 ([5]). Let $\rho$ be a function modular satisfying the $\Delta_{2}$-condition and let $B$ be a $\|\cdot\|_{\rho}$-closed subset of $L_{\rho}$. Let $k \in(0,1)$ and $T: B \rightarrow B$ be a single-valued mapping such that

$$
\rho(T(f)-T(g)) \leq k \rho(f-g)
$$

where $f, g \in B$. Then $T$ has a fixed point if $\sup _{n}\left(2 T^{n}\left(f_{0}\right)\right)<1$.

Since then, there has been significant work on extending and improving this results further in.

Ait Taleb and Hanebaly presented some examples proving that the Theorem 2 (Theorem I-1 of [42]) tends to be more applicable than Theorem 1. However, Theorem 1 is not generalized by Theorem 2 .

Theorem 2 ([42]). Suppose that $X_{\rho}$ is a $\rho$-complete modular space where $\rho$ is an $\tilde{s}$-convex modular satisfying the $\Delta_{2}$-condition and has the Fatou property. Moreover, assume that $B$ is a $\rho$-closed 
subset of $X_{\rho}$ and $T: B \rightarrow B$ is a single-valued mapping such that for some $c, k \in \mathbb{R}^{+}$and $c>\max \{1, k\}$ satisfies

$$
\rho(c(T(x)-T(y))) \leq k^{\tilde{s}} \rho(x-y),
$$

where $x, y \in B$. Then $T$ has a fixed point.

As mentioned in [42], we can ask what if it is mentioned that they are unable to prove whether the conclusion of Theorem 2 is true, if we have $c=1$ and $0<k<1$. We note that our main result, Theorem 8 , has replied to this open question, also it has generalized Theorem 1, when $w_{\rho}(2)<+\infty$.

Some extensions were subsequently proposed by various authors, taking into account different relaxations that require. A new extension of Theorem 1, based on the cases of correspondences, appeared in 2006 in Theorem 3-1 of [9] as follows.

Theorem 3 ([9]). Let $\rho$ be a convex modular satisfying the $\Delta_{2}$-type condition, $B$ be a nonempty $\rho$-bounded $\rho$-closed subset of $L_{\rho}$, and $f: B \rightarrow B$ be a $\rho$-closed valued correspondence such that there exists a constant $k \in[0,1)$ that

$$
H_{\rho}\left(f\left(f_{1}\right), f\left(f_{2}\right)\right) \leq k \rho\left(f_{1}-f_{2}\right),
$$

where $f_{1}, f_{2} \in B$. Then $f$ has a fixed point.

Later, in 2009, this result is improved to Theorem 2-1 in [13] by the following result.

Theorem 4 ([13]). Let $\rho$ be a convex modular satisfying $\Delta_{2}$-type condition and $B \subset L_{\rho}$ be a nonempty $\rho$-closed $\rho$-bounded subset of the modular space $L_{\rho}$. Then any closed valued correspondence $f: B \rightarrow B$ such that for $f_{1}, f_{2} \in B$ and $f_{3} \in f\left(f_{1}\right)$, there is $f_{4} \in f\left(f_{2}\right)$ such that

$$
\rho\left(f_{3}-f_{4}\right) \leq k \rho\left(f_{2}-f_{1}\right),
$$

where $k \in(0,1)$, has a fixed point.

In both Theorems 3 and 4, it is assumed that the correspondence is defined on a $\rho$-bounded subset of a modular space $(X, \rho)$ with a convex modular. In [32] Theorem 2-5, the correspondence has $\rho$-compact values.

Theorem 5 ([32]). Let $B$ be a $\rho$-bounded subset of a $\rho$-complete space $(X, \rho)$. Let $f: B \rightarrow B$ be a correspondence with $\rho$-compact values that for each $x, y \in C$ and $z \in f(x)$, there exists $w \in f(y)$ such that

$$
\rho(z-w) \leq k \rho(x-y)
$$

where $2 k w_{\rho}(2)^{2}<1$. Then $f$ has a fixed point.

Our main result, Theorem 8 , is a generalization of Theorems 3-5.

The main result of [15] is the following theorem.

Theorem 6 ([15]). Let $(X, \perp, d)$ be an O-complete metric space. And let $T: X \rightarrow X$ be $\perp$ continuous mapping, $\perp-$ Banach contraction and $\perp$-preserving. Then, $T$ has a unique fixed point $z \in X$. Also, $T$ is a Picard operator, that is, $\lim _{n \rightarrow+\infty} T^{n}(x)=z$ for all $x \in X$.

In [16], the Meir-Keeler condition on orthogonal modular space was introduced and the Meir-Keeler Theorem on orthogonal modular spaces was proven, see Theorem 7. 
Definition 12 ([16]). Let $(X, \perp, \rho)$ be an orthogonal modular space. Let $B$ be an SO-closed subset of $X_{\rho}$ and $c, l \in(0,+\infty)$ with $c>l$. We say that a mapping $T: B \rightarrow B$ satisfies the Meir-Keeler condition whenever for every $\epsilon>0$, there exists $\delta(\epsilon)>0$ such that:

$$
x \neq y, \quad x \perp y \text { and } \epsilon \leq \rho(l(x-y))<\epsilon+\delta(\epsilon) \text { implies } \rho(c(T x-T y))<\epsilon .
$$

Theorem 7 ([16]). Let $(X \perp, \rho)$ be an SO-complete orthogonal modular space with an orthogonal element $x_{0}$ and $\rho$ satisfies the $\Delta_{2}$-condition. Suppose that $B$ is an $S O$-closed subset of $X_{\rho}$ such that $x_{0} \in B$ and there exist $c, l \in(0,+\infty)$ with $c>l$. Assume that $T: B \rightarrow B$ is orthogonal preserving, SO-continuous such that satisfying the Meir-Keeler condition. Then, $T$ has a unique fixed point $z \in B$. Also, $T$ is a Picard operator, that is, for all $x \in B$, the sequence $\left(T^{n}(x)\right)$ is $\rho$-convergent to $z$.

Then, in the following sections, we provide some conditions for guarantee the existence of fixed points for myriad mappings and some strong assumptions such as the convexity of the modular and the $\rho$-boundedness of the domain of the correspondence are relaxed, also the $\rho$-completeness is replaced by $S O$-complete which can lead to making our theorems much stronger and more applicable.

\section{Main Results}

In this section, we focus on an $S O$-complete space $X$ (not necessarily $\rho$-complete modular space) and consider the orthogonal preserving correspondence $f: X \rightarrow X$ with $S O-$ closed valued. Further, we assume $\omega_{\rho}(2)<+\infty$. Also, in order to simplify notation, let us denote $X=(X, \rho)$.

The following lemma is a handy tool that will be used in the sequel. By the definition, every orthogonal modular space is a modular space too, so the following lemma remains correct for an orthogonal modular space and we will use it in our results.

Lemma 1 ([43]). A sequence $\left(x_{n}\right)$ in a modular space $(X, \rho)$ is a $\rho$-Cauchy sequence if there exists $k \in[0,1)$ such that

$$
\rho\left(x_{n}-x_{n+1}\right) \leq k \rho\left(x_{n-1}-x_{n}\right)
$$

for every $n \in \mathbb{N}$.

The following theorem is an equivalent of Nadler's theorem [8] on an orthogonal modular space.

Theorem 8. Consider $k \in[0,1)$ and for every $x, y \in X$, such that $x \perp y$, there exists $w \in f(y)$ such that $\rho(z-w) \leq k \rho(x-y)$ for every $z \in f(x)$. Then SOUHC mapping $f$ has a fixed point.

Proof. Suppose that $x_{0} \in X$ is an orthogonal element and $x_{1} \in f\left(x_{0}\right)$, so $x_{0} \perp x_{1}$. We know from our assumption that there exists $x_{2} \in X$ such that $x_{2} \in f\left(x_{1}\right)$ and

$$
\rho\left(x_{2}-x_{1}\right) \leq k \rho\left(x_{1}-x_{0}\right)
$$

Since $f$ is orthogonal preserving, we have $x_{1} \perp x_{2}$. By the same way, for every $n \geq 1$, we have $x_{n-1} \perp x_{n}$, so by the contraction, there exists $x_{n+1} \in X$ such that $x_{n+1} \in f\left(x_{n}\right)$ and

$$
\rho\left(x_{n+1}-x_{n}\right) \leq k \rho\left(x_{n}-x_{n-1}\right) .
$$

Note that by Lemma $1,\left(x_{n}\right)$ is a Cauchy $S O$-sequence in the $S O$-complete space $X$. Which means that there exists $x \in X$ such that $x_{n} \rightarrow x$ as $n \rightarrow+\infty$. Since $f$ is SOUHC and $x_{n} \in f\left(x_{n-1}\right)$, there exists a subsequence of $x_{n}$ which is convergent to $z \in f(x)$. Since $f$ is $S O$-closed, and the SO-sequence $x_{n}$ is $\rho$-convergent to $x$. This implies that $z=x$, so $x \in f(x)$. 
The following theorem generalizes Theorem 8 .

Theorem 9. Consider for every $x, y \in X$ that $x \perp y$ and $z \in f(x)$, there exists $w \in f(y)$ such that

$$
\rho(z-w) \leq k \max \{\rho(x-y), \alpha \rho(x-z), \alpha \rho(y-w)\},
$$

where $\alpha \in[0,1]$, and $k \in[0,1)$. Then $f$ has a fixed point if one of the following assumptions satisfies:

(i) $f$ is SOUHC.

(ii) $\rho$ is continuous i.e., $\lim \rho\left(x_{n}\right)=\rho(x)$ as $x_{n} \rightarrow x$.

Proof. Let us define a sequence $\left(x_{n}\right)$ that $x_{0}$ is an orthogonal element, $x_{1} \in f\left(x_{0}\right)$ and SO-sequence $x_{n+1} \in f\left(x_{n}\right)$ such that

$$
\rho\left(x_{n+1}-x_{n}\right) \leq k \max \left\{\rho\left(x_{n}-x_{n-1}\right), \alpha \rho\left(x_{n}-x_{n-1}\right), \alpha \rho\left(x_{n+1}-x_{n}\right)\right\},
$$

for every $n \geq 1$. As it becomes clear by Equation (1), the right hand side of this equation is not $\alpha \rho\left(x_{n}-x_{n+1}\right)$ or $\alpha \rho\left(x_{n}-x_{n-1}\right)$. Now it is easy to see that

$$
\rho\left(x_{n+1}-x_{n}\right) \leq k \rho\left(x_{n}-x_{n-1}\right)
$$

Thus it follows from Lemma 1 and SO-completeness of $X$ that there exists $x \in X$ such that $x_{n} \rightarrow x$ as $n \rightarrow \infty$.

The proof is obviously complete under assumption $(i)$. Now assume $(i i)$ holds. Let $y_{n} \in f(x)$, since $\rho$ is continuous, we have $\lim _{n \rightarrow+\infty} \rho\left(x_{n}-y_{n}\right)=\lim _{n \rightarrow+\infty} \rho\left(x-y_{n}\right)=0$. Now from the facts that $f(x)$ is $S O$-closed, $x \in f(x)$.

Khan et. al. (in [44]) introduced the notion of altering distance function defined in the following way.

Definition 13 ([44]). A function $\psi: \mathbb{R}^{+} \rightarrow \mathbb{R}^{+}$is called an altering distance function if the following conditions hold.

(1) $\psi$ is continuous and nondecreasing,

(2) $\psi(t)=0$ if and only if $t=0$.

Theorem 10. Suppose that there exists $w \in f(y)$ such that for all $x, y \in X$, that $x \perp y$

$$
\psi(\lambda \rho(z-w)) \leq \psi(S(x, y))+L \psi(I(x, y))
$$

where constants $L \geq 0, \lambda>1$, also

$$
S(x, y)=\max \left\{\rho(x-y), \operatorname{dist}_{\rho}(x, f(x)), \operatorname{dist}_{\rho}(y, f(y)) \frac{1+\operatorname{dist}_{\rho}(x, f(x))}{1+\rho(x-y)}\right\}
$$

and

$$
I(x, y)=\min \left\{\operatorname{dist}_{\rho}(x, f(x))+\operatorname{dist}_{\rho}(y, f(y)), \operatorname{dist}_{\rho}(x, f(y)), \operatorname{dist}_{\rho}(y, f(x))\right\} .
$$

and $z \in f(x)$. Then $f$ has a fixed point if and only if one of the following assumptions holds.

(i) $f$ is weakly Picard.

(ii) $f$ is SOUHC.

(iii) $\rho$ is continuous. 
Proof. Choose an orthogonal element $x_{0} \in X$ then find $x_{1} \in f\left(x_{0}\right)$, continuing on the same lines we can construct a sequence $x_{n}$ such that $x_{n+1} \in f\left(x_{n}\right)$ that $x_{n-1} \perp x_{n}$ and

$$
\psi\left(\lambda \rho\left(x_{n}-x_{n+1}\right)\right) \leq \psi\left(S\left(x_{n-1}, x_{n}\right)\right)+L \psi\left(I\left(x_{n-1}, x_{n}\right)\right),
$$

for every $n \geq 1$. From (5), $I\left(x_{n-1}, x_{n}\right)=0$. Now, from (4), we have

$$
\begin{aligned}
S\left(x_{n-1}, x_{n}\right)= & \max \left\{\rho\left(x_{n-1}-x_{n}\right), \operatorname{dist}_{\rho}\left(x_{n-1}, f\left(x_{n-1}\right),\right.\right. \\
& \left.\operatorname{dist}_{\rho}\left(x_{n}, f\left(x_{n}\right)\right) \frac{1+\operatorname{dist}_{\rho}\left(x_{n-1}, f\left(x_{n-1}\right)\right.}{1+\rho\left(x_{n-1}-x_{n}\right)}\right\}, \\
\leq & \max \left\{\rho\left(x_{n-1}-x_{n}\right), \rho\left(x_{n-1}-x_{n}\right),\right. \\
& \left.\rho\left(x_{n}-x_{n+1}\right) \frac{1+\rho\left(x_{n-1}-x_{n}\right)}{1+\rho\left(x_{n-1}-x_{n}\right)}\right\}, \\
= & \max \left\{\rho\left(x_{n}-x_{n+1}\right), \rho\left(x_{n-1}-x_{n}\right)\right\} .
\end{aligned}
$$

If $\max \left\{\rho\left(x_{n}-x_{n+1}\right), \rho\left(x_{n-1}-x_{n}\right)\right\}=\rho\left(x_{n}, x_{n+1}\right)$ then using inequality (3) we have

$$
\psi\left(\lambda \rho\left(x_{n}-x_{n+1}\right)\right) \leq \psi\left(\rho\left(x_{n}-x_{n+1}\right)\right)+L \psi(0) .
$$

Since $\psi(0)=0$ and $\psi$ is nondecreasing. Then above inequality can be written as

$$
\lambda \rho\left(x_{n}-x_{n+1}\right) \leq \rho\left(x_{n}-x_{n+1}\right),
$$

which is a contradiction because $\lambda>1$.

Hence $\max \left\{\rho\left(x_{n}-x_{n+1}\right), \rho\left(x_{n-1}-x_{n}\right)\right\}=\rho\left(x_{n-1}-x_{n}\right)$.

Then inequality (3) becomes $\psi\left(\lambda \rho\left(x_{n}-x_{n+1}\right)\right) \leq \psi\left(\rho\left(x_{n-1}-x_{n}\right)\right)$.

So, we have $\lambda \rho\left(x_{n}-x_{n+1}\right) \leq \rho\left(x_{n-1}-x_{n}\right)$, for all $n \in \mathbb{N}$. Now, using Lemma (1) and SO-completeness of $B$, there exists $x \in B$ such that $x_{n} \rightarrow x$ as $n \rightarrow+\infty$.

The proof is obviously complete under assumption (i), (ii) and (iii).

\section{Open Questions}

Radenović et. al. in [45] considered the following open problem:

Problem 1. If $T: B \rightarrow B$ is a single-valued mapping such that

$$
\rho(T(x)-T(y)) \leq k \max \{\rho(x-y), \rho(x-T(x)), \rho(y-T(y)), \rho(x-T(y)), \rho(y-T(x))\},
$$

for every $x, y \in B$ where $B \subseteq X$ and $k \in \mathbb{R}^{>0}$, then under what constraints does $T$ have $a$ fixed point?

It can be answered under the constraints that $T: B \rightarrow B$ is a single-valued mapping and $k \in\left(0, \frac{1}{w_{\rho}(2)\left(1+w_{\rho}(2)\right)}\right)$. However, there is no answer to this question in the case of the correspondence $f$ or $k \geq \frac{1}{w_{\rho}(2)\left(1+w_{\rho}(2)\right)}$, with the matched definitions for correspondences.

Existence of a fixed point for $T$ has been successfully shown if $k \in\left(0, \frac{1}{w_{\rho}(2)\left(1+w_{\rho}(2)\right)}\right)$ (given by Radenović et al. in [45]). Interestingly, this question can be reformulated in the next theorem and we prove it for correspondences with $k \in\left[0, \frac{1}{2 w_{\rho}(2)}\right]$.

Theorem 11. Let $f$ be an SOUHC correspondence that for each $x, y \in X$ that $x \perp y$ and $z \in f(x)$, there exists $w \in f(y)$ such that

$$
\rho(z-w) \leq k \max \{\rho(x-y), \rho(x-z), \rho(y-w), \rho(x-w), \rho(y-z)\},
$$

where $2 k w_{\rho}(2)<1$. Then $f$ has a fixed point. 
Proof. Let $x_{0}$ be an orthogonal element and $x_{1} \in f\left(x_{0}\right)$ for an arbitrary $x_{0} \in X$. By assumption, for every $n \geq 1$ there exists $x_{n+1} \in f\left(x_{n}\right)$ such that $x_{n} \perp x_{n+1}$ and

$$
\rho\left(x_{n+1}-x_{n}\right) \leq k \max \left\{\rho\left(x_{n}-x_{n-1}\right), \rho\left(x_{n+1}-x_{n}\right), \rho\left(x_{n-1}-x_{n+1}\right), \rho\left(x_{n}-x_{n}\right)\right\} .
$$

It follow that

$$
\begin{aligned}
\rho\left(x_{n+1}-x_{n}\right) & \leq k \max \left\{\rho\left(x_{n}-x_{n-1}\right), \rho\left(x_{n+1}-x_{n}\right), w_{\rho}(2)\left(\rho\left(x_{n-1}-x_{n}\right)+\rho\left(x_{n}-x_{n+1}\right)\right)\right\}, \\
& \leq k w_{\rho}(2)\left(\rho\left(x_{n-1}-x_{n}\right)+\rho\left(x_{n}-x_{n+1}\right)\right),
\end{aligned}
$$

which implies that $\rho\left(x_{n+1}-x_{n}\right) \leq k^{\prime} \rho\left(x_{n-1}-x_{n}\right)$, where $k^{\prime}=\frac{k w_{\rho}(2)}{1-k w_{\rho}(2)}$. Note that $k^{\prime}$ is not greater than one, since $k<\frac{1}{2 w_{\rho}(2)}$. In addition, from the fact that $X$ is a $S O-$ complete set and $x_{n}$ is a Cauchy $S O-$ sequence by Lemma 1 , there exists $x \in X$ such that $x_{n} \rightarrow x$. From SOUHC, we have $x \in f(x)$.

\section{Application to Integral Inclusions}

A modular fixed point theorem can be used for providing sufficient (but not necessary) conditions for existence of a real continuous function $u$ defined on $[a, b]$ such that

$$
u(t)=v(t)+\gamma \int_{a}^{b} G(t, s) g(s, u(s)) d s, \quad t \in[a, b],
$$

where $\gamma$ is a constant, $g:[a, b] \times \mathbb{R} \rightarrow[a, b]$ is OUHC, $G:[a, b] \times[a, b] \rightarrow[0,+\infty)$ and $v:[a, b] \rightarrow \mathbb{R}$ are given continuous functions.

For simplicity, we introduce the following shorthand notations. We use $X=C[a, b]$ to denote all real continuous functions defined on $[a, b], g_{u}:[a, b] \rightarrow[a, b]$, where $g_{u}(s)=$ $g(s, u(s))$ and a modular $\rho$ defined on $X$ as

$$
\rho(u)=\max _{a \leq t \leq b}|u(t)|^{2} .
$$

It is not difficult to prove that $(X, \rho)$ is a $\rho$-complete modular space. Now the aforementioned integral inclusion problem (9) can be reformulated as $u$ and is a solution of problem (9) if and only if it is a fixed point of $f: X \rightarrow X$ defined as

$$
f(u)=\left\{x \in X: x(t) \in v(t)+\gamma \int_{a}^{b} G(t, s) g(s, u(s)) d s\right\} .
$$

Now, we show under the following mild assumptions,

1. $|\gamma| \leq 1$,

2. $\max _{a \leq t \leq b} \int_{a}^{b} G^{2}(t, z) d z \leq \frac{1}{b-a}$,

3. for all $x, y \in X$ and $w_{x}(t) \in g_{x}(t)$, there exists $h_{y}(t) \in g_{y}(t)$ such that

$$
\left|w_{x}(t)-h_{y}(t)\right|^{2} \leq \frac{1}{s}|x(t)-y(t)|^{2}, t \in[a, b]
$$

where $s>1$,

the correspondence $f$ has a unique fixed point. So, we assume that $x, y \in X$ and $w \in f(x)$ by definition, we have

$$
w(t)=v(t)+\gamma \int_{a}^{b} G(t, s) g(s, x(s)) d s
$$


By Michael's selection theorem, (in [46] Theorem 1), it follows that there exists a continuous single- valued mapping $w_{x}(s) \in g_{x}(s)$ that $w(t)=v(t)+\gamma \int_{a}^{b} G(t, s) w_{x}(s) d s$. According to assumption 3, for $w_{x}(s) \in g_{x}(s)$, there is an $h_{y}(s) \in g_{y}(s)$ such that

$$
\left|w_{x}(s)-h_{y}(s)\right|^{2} \leq \frac{1}{s}|x(s)-y(s)|^{2} .
$$

We define

$$
h(t)=v(t)+\gamma \int_{a}^{b} G(t, s) h_{y}(s) d s
$$

i.e.,

$$
h(t) \in v(t)+\gamma \int_{a}^{b} G(t, s) g_{y}(s) d s .
$$
we have

Therefore $h \in f(y)$. Using the Cauchy-Schwarz inequality and conditions $1-3$,

$$
\begin{aligned}
\rho(w-h) & =\max _{a \leq t \leq b}|w(t)-h(t)|^{2}, \\
& =\max _{a \leq t \leq b}\left|v(t)+\gamma \int_{a}^{b} G(t, s) w_{x}(s) d s-\left(v(t)+\gamma \int_{a}^{b} G(t, s) h_{y}(s) d s\right)\right|^{2}, \\
& =|\gamma|^{2} \max _{a \leq t \leq b}\left|\int_{a}^{b} G(t, s)\left(w_{x}(s)-h_{y}(s)\right) d s\right|^{2}, \\
& \leq|\gamma|^{2} \max _{a \leq t \leq b}\left\{\int_{a}^{b} G^{2}(t, s) d s \int_{a}^{b}\left|w_{x}(s)-h_{y}(s)\right|^{2} d s\right\} \\
& =|\gamma|^{2}\left\{\max _{a \leq t \leq b} \int_{a}^{b} G^{2}(t, s) d s\right\} \cdot\left\{\int_{a}^{b}\left|w_{x}(s)-h_{x}(s)\right|^{2} d s\right\} \\
& \leq \frac{|\gamma|^{2}}{b-a}\left\{\frac{1}{s} \int_{a}^{b}|x(s)-y(s)|^{2} d s\right\} \\
& \leq \frac{|\gamma|^{2}}{s(b-a)} \int_{a}^{b} \max _{a \leq s \leq b}|x(s)-y(s)|^{2} d s, \\
& =\frac{|\gamma|^{2}}{s} \max _{a \leq s \leq b}|x(s)-y(s)|^{2}, \\
& =\frac{1}{s} \rho(x-y) .
\end{aligned}
$$

Theorem 8 implies that $f$ has a unique fixed point $u \in X$, that is, the integral inclusion (9) has a solution which belongs to $C[a, b]$.

\section{Conclusions}

In this paper, we defined some contractive correspondences and obtained fixed point results in orthogonal modular spaces. As a consequence of our main result, we obtained the Banach contraction principle in the orthogonal modular space for correspondences. Also, we improved the interval of contraction (proposed in an open problem) which shows that our results are still valid if we choose contractive constant $k$ in $(0,1)$. As an application of our result, we obtained a solution to integral inclusions in the orthogonal modular space. Further, we provide examples that further elaborate the usability of the obtained results. It is worth mentioning that we identify the relationship between orthogonal modular spaces and modular spaces which will be helpful to prove various results in orthogonal modular spaces.

Author Contributions: Conceptualization, F.L., N.S., L.G. and M.F.B.; Formal analysis, F.L. and N.S.; Investigation, L.G.; Methodology, N.S. and L.G.; Supervision, M.F.B.; Validation, F.L.; Writingoriginal draft, F.L. and N.S.; Writing-review-editing, L.G. and M.F.B. All authors contributed equally. All authors have read and agreed to the published version of the manuscript. 
Funding: This research received no external funding.

Institutional Review Board Statement: Not applicable.

Informed Consent Statement: Not applicable.

Data Availability Statement: Not applicable.

Conflicts of Interest: The authors declare no conflict of interest.

\section{References}

1. Banach, S. Sur les opérations dans les ensembles abstraits et leur application aux équations intégrales. Fund. Math. 1922, 3, 133-181. [CrossRef]

2. Musielak, J.; Orlicz, W. On modular spaces. Stud. Math. 1959, 18, 49-65. [CrossRef]

3. Musielak, J. Orlicz Spaces and Modular Spaces; Springer: Berlin, Germany, 1983; pp. 1-216.

4. Nakano, H. Modulared Semi-Ordered Linear Spaces; Maruzen Co., Ltd.: Tokyo, Japan, 1950.

5. Khamsi, M.A.; Kozlowski, W.M.; Reich, S. Fixed point theory in modular function spaces. Nonlinear Anal. 1990, 14, 935-953. [CrossRef]

6. Khamsi, M.A. A convexity property in modular function spaces. Math. Jpn. 1996, 44, 269-280.

7. Khamsi, M.A.; Kozlowski, W.M.; Shutao, C. Some geometrical properties and fixed point theorems in Orlicz spaces. J. Math. Anal. Appl. 1991, 155, 393-412. [CrossRef]

8. Nadler, S.B. Multi-valued contraction mappings. Pac. J. Math. 1969, 30, 475-488. [CrossRef]

9. Dhompongsa, S.; Benavides, T.; Kaewcharoen, A.; Panyanak, B. Fixed point theorems for multivalued mappings in modular function spaces. Sci. Math. Jpn. 2006, 2006, 139-147.

10. Feng, Y.; Liu, S. Fixed point theorems for multi-valued contractive mappings and multivalued Caristi type mappings. J. Math. Anal. Appl. 2006, 317, 103-112. [CrossRef]

11. Klim, D.; Wardowski, D. Fixed point theorems for set-valued contractions in complete metric spaces. J. Math. Anal. Appl. 2007, 334, 132-139. [CrossRef]

12. Petruşel, A.; Petruşel, G. On Reich's strict fixed point theorem for multi-valued operators in complete metric spaces. J. Nonlinear Var. Anal. 2018, 2, 103-112.

13. Kutbi, M.A.; Latif, A. Fixed points of multivalued maps in modular function spaces. Fixed Point Theory Appl. 2009, $2009,1-12$. [CrossRef]

14. Alfuraidan, M.R. Fixed points of multivalued mappings in modular function spaces with a graph. Fixed Point Theory Appl. 2015, 1, 1-14. [CrossRef]

15. Eshaghi, M.; Ramezani, M.; De La Sen, M.; Cho, Y.J. On orthogonal sets and Banach's fixed point theorem. Fixed Point Theory 2017,18, 569-578.

16. Ramezani, M.; Baghani, H. The Meir-Keeler fixed point theorem in incomplete modular spaces with application. J. Fixed Point Theory Appl. 2017, 19, 2369-2382. [CrossRef]

17. Van Dung, N. Notes on orthogonal-complete metric spaces. Bull. Aust. Math. Soc. 2021, 1-7. [CrossRef]

18. Baghani, H.; Eshaghi Gordji, M.; Ramezani, M. Orthogonal sets, The axiom of choice and proof of a fixed point theorem. J. Fixed Point Theory Appl. 2016, 18, 465-477. [CrossRef]

19. Eivazi Damirchi Darsi Olia, Z.; Eshaghi, M.; Ebrahimi Bagha, D. Banach fixed point theorem on orthogonal cone metric spaces. Facta Univ. Ser. Math. Inform. 2020, 35, 1239-1250.

20. Hosseini, H.; Eshaghi, M. Fixed Point Results in Orthogonal Modular Metric Spaces. Int. J. Nonlinear Anal. Appl. 2020, 11, 425-436.

21. Alaca, C.; Ege, M.E.; Park, C. Fixed point results for modular ultrametric spaces. J. Comput. Anal. Appl. 2016, 20, $1259-1267$.

22. Bakhtin, I.A. The contraction mapping principle in almost metric spaces. Funct. Anal. 1989, 30, $26-37$.

23. Chaipunya, P.; Mongkolkeha, C.; Sintunavarat, W.; Kumam, P. Fixed point theorems for multivalued mappings in modular metric spaces. Abstr. Appl. Anal. 2012, 2012, 503504.

24. Kozlowski, W.M. Notes on modular function spaces-II. Comment. Math. 1988, 28, 101-116.

25. Nourouzi, K.; Shabanian, S. Operators defined on n-modular spaces. Mediterr. J. Math. 2009, 6, 431-446. [CrossRef]

26. Parvaneh, V.; Hussain, N.; Khorshidi, M.; Mlaiki, N.; Aydi, H. Fixed Point Results for Generalized $\mathfrak{F}-$ Contractions in Modular $b$-Metric Spaces with Applications. Mathematics 2019, 7, 887. [CrossRef]

27. Petruşel, A. Local fixed point results for graphic contractions. J. Nonlinear Var. Anal. 2019, 3, 141-148.

28. Shabanian, S. Modular Space and Fixed Point Theorems. Ph.D. Thesis, K.N.Toosi University of Technology, Tehran, Iran, 2007.

29. Orlicz, W. Über eine gewisse klasse von Raumen vom Typus B. Bull. Acad. Polon. Sci. A 1932, 207-220.

30. Orlicz, W. Über Raumen $L^{M}$. Bull. Acad. Polon. Sci. A 1936, 93-107.

31. Benavides, T.; Khamsi, M.A.; Samadi, S. Asymptotically regular mappings in modular function spaces. Sci. Math. Jpn. 2001, 4, 239-248.

32. Lael, F.; Nourouzi, K. On the fixed points of correspondences in modular spaces. Int. Sch. Res. Not. 2011, 2011, 530254. [CrossRef]

33. Eshaghi, M.; Habibi, H. Existence and uniqueness of solutions to a first-order differential equation via fixed point theorem in orthogonal metric space. Facta Univ. Ser. Math. Inform. 2019, 34, 123-135. 
34. Eshaghi, M.; Habibi, H. Fixed point theory in $\varepsilon$-connected orthogonal metric space. Sahand Commun. Math. Anal. 2019, 16, 35-46.

35. Eshaghi, M.; Habibi, H.; Sahabi, M.B. Orthogonal sets; orthogonal contractions. Asian-Eur. J. Math. 2019, 12, 1950034. [CrossRef]

36. Eshaghi, M.; Habibi, H. Fixed point theory in generalized orthogonal metric space. J. Linear And Topol. Algebra 2017, 6, 251-260.

37. Kassu, W.W.; Sangago, M.G.; Zegeye, H. Convergence theorems to common fixed points of multi-valued $\rho$-quasi-nonexpansive mappings in modular function spaces. Adv. Fixed Point Theory 2018, 8, 21-36.

38. Khan, S.H. Approximating fixed point of $(\lambda, \rho)$-firmly nonexpansive mappings in modular function spaces. Arab. J. Math. 2018, 7, 281-287. [CrossRef]

39. Japón, M.A. Some geometric properties in modular spaces and application to fixed point theory. J. Math. Anal. Appl. 2004, 295, 576-594. [CrossRef]

40. Brodskii, M.S.; Milman, D.P. On the center of a convex set (Russian). Dokl. Akad. Nauk. SSSR 1948, 59, 837-840.

41. Japon, M.A. Applications of Musielak-Orlicz spaces in modern control systems. Teubner-Texte Math. 1988, 103, 34-36.

42. Taleb, A.; Hanebaly, E. A fixed point theorem and its application to integral equations in modular function spaces. Proc. Am. Math. Soc. 2000, 128, 419-426. [CrossRef]

43. Lael, F.; Shabanian, S. Convexity and boundedness relaxation for fixed point theorems in modular spaces. Appl. Gen. Topol. 2021, 22, 91-108. [CrossRef]

44. Khan, M.S.; Swaleh, M.; Sessa, S. Fixed point theorems by altering distances between the points. Bull. Aust. Math. Soc. 1984, 30, 1-9. [CrossRef]

45. Radenović, S.; Došenović, T.; Lampert, T.A.; Golubovć, Z. A note on some recent fixed point results for cyclic contractions in $b$-metric spaces and an application to integral equations. Appl. Math. Comput. 2016, 273, 155-164. [CrossRef]

46. Miculescu, R.; Mihail, A. New fixed point theorems for set-valued contractions in $b$-metric spaces. J. Fixed Point Theory Appl. 2017, 19, 2153-2163. [CrossRef] 